\title{
Stage 0 Intrahepatic Cholangiocarcinoma AJCC v7
}

National Cancer Institute

\section{Source}

National Cancer Institute. Stage O Intrahepatic Cholangiocarcinoma A/CC v7. NCI

Thesaurus. Code C88049.

Stage 0 includes: T is, NO, MO. T is: Carcinoma in situ (intraductal tumor). N0: No regional lymph node metastasis. M0: No distant metastasis. (from AJCC 7th Ed.) 